\title{
DEPTH AND STANLEY DEPTH OF MULTIGRADED MODULES
}

\author{
ASIA RAUF
}

\begin{abstract}
We study the behavior of depth and Stanley depth along short exact sequences of multigraded modules and under reduction modulo an element.
\end{abstract}

\section{INTRODUCTION}

Let $K$ be a field and $S=K\left[x_{1}, \ldots, x_{n}\right]$ a polynomial ring in $n$ variables over $K$. Let $M$ be a finitely generated multigraded (i.e. $\mathbb{Z}^{n}$-graded) $S$-module. Let $m \in M$ be a homogeneous element in $M$ and $Z \subseteq\left\{x_{1}, \ldots, x_{n}\right\}$. We denote by $m K[Z]$ the $K$-subspace of $M$ generated by all elements $m v$, where $v$ is a monomial in $K[Z]$. The multigraded $K$-subspace $m K[Z] \subset M$ is called Stanley space of dimension $|Z|$, if $m K[Z]$ is a free $K[Z]$ module. A Stanley decomposition of $M$ is a presentation of the $K$-vector space $M$ as a finite direct sum of Stanley spaces $\mathcal{D}: M=\bigoplus_{i=1}^{r} m_{i} K\left[Z_{i}\right]$. Set $\operatorname{sdepth} \mathcal{D}=\min \left\{\left|Z_{i}\right|\right.$ : $i=1, \ldots, r\}$. The number

$$
\operatorname{sdepth}(M):=\max \{\operatorname{sdepth}(\mathcal{D}): \mathcal{D} \text { is a Stanley decomposition of } M\}
$$

is called Stanley depth of $M$. In 1982, Richard P. Stanley [14, Conjecture 5.1] conjectured that $\operatorname{sdepth}(M) \geq \operatorname{depth}(M)$ for all finitely generated $\mathbb{Z}^{n}$-graded $S$-modules $M$. The conjecture is discussed in some special cases in [7, [2, [9], [13], [1, [12] [4], [5].

If $M$ is a finitely generated module over a Noetherian local ring $(R, m)$ and $x \in m$ then it is well-known that $\operatorname{dim} M / x M \geq \operatorname{dim} M-1$. Our Proposition 1.2 and Lemma 1.7, show that the above inequality is preserved for depth and sdepth when $M=S / I$ and $I \subset S$ is a monomial ideal and $x=x_{k}$ for any $k \in[n]$. If $M$ is a general multigraded $S$-module, then we might have depth $M / x_{k} M<\operatorname{depth} M-1$ as shows Example 1.5. Also we might have sdepth $M / x_{k} M<\operatorname{sdepth} M-1$ even if $x_{k}$ is regular on $M$, as shows Example 1.8,

As we know depth decreases by one if we reduce modulo a regular element. In [13, Theorem 1.1], it is proved that the corresponding statement holds for the Stanley depth in the case of $M=S / I$ where $I \subset S$ is a monomial ideal and $f$ a monomial in $S$ which is regular on $M$. The next question arises whether this is true for any multigraded module? The answer is no, see Example 1.8, Let

$$
\mathcal{F}: 0=M_{0} \subset M_{1} \subset \ldots \subset M_{r}=M
$$

be a chain of $\mathbb{Z}^{n}$-graded submodules of $M$. Then $\mathcal{F}$ is called a prime filtration of $M$ if $M_{i} / M_{i-1} \cong\left(S / P_{i}\right)\left(-a_{i}\right)$ where $a_{i} \in \mathbb{Z}^{n}$ and $P_{i}$ is a monomial prime ideal for all $i$. We denote $\operatorname{Supp} \mathcal{F}=\left\{P_{1}, \ldots, P_{r}\right\}$. A finitely generated module $M$ is called almost clean if there exists a prime filtration $\mathcal{F}$ of $M$ such that $\operatorname{Supp}(\mathcal{F})=\operatorname{Ass}(M)$. We show in Lemma 1.9 that for almost clean module $M$ and $x_{k} \in S$ being regular on $M$, we have sdepth $M / x_{k} M \geq \operatorname{sdepth} M-1$. However, we show in Proposition 1.10 that

1991 Mathematics Subject Classification. Primary 13H10, Secondary 13P10, 13C15, 13 F20.

Key words and phrases. Multigraded modules, depth, Stanley decompositions, Stanley depth.

The author wants to acknowledge Higher Education Commission Pakistan for partial financial support during preparation of this work. 
sdepth $M / x_{k} M \leq \operatorname{sdepth} M-1$, if $x_{k}$ is regular on $M$. As an application we get that Stanley's conjecture holds for $M$ if it holds for the module $M / x_{k} M$ (see Corollary 1.11). Moreover if $M$ has a maximal regular sequence given by monomials then Stanley's conjecture holds for $M$ (see Corollary 1.13).

Given a short exact sequence of finitely generated multigraded $S$-modules, then the Stanley depth of the middle one is greater than or equal to the minimum of Stanley depths of the ends (see Lemma 2.2). Several examples show that the "Depth lemma" is mainly wrong in the frame of sdepth (see Examples 2.5 and 2.6). However, we prove in Lemma 2.7 that if $I$ is any monomial complete intersection of $S$, then sdepth $I$ is greater than or equal to sdepth $S / I+1$. But in general for any monomial ideal this inequality is still an open question.

In the last section, we prove that if $I \subset S_{1}=K\left[x_{1}, \ldots, x_{n}\right], J \subset S_{2}=K\left[y_{1}, \ldots, y_{m}\right]$ are monomial ideals and $S=K\left[x_{1}, \ldots, x_{n}, y_{1}, \ldots, y_{m}\right]$, then the Stanley depth of the tensor product of $S_{1} / I$ and $S_{2} / J$ (over $K$ ) is greater than or equal to the sum of $\operatorname{sdepth} S_{1} / I$ and sdepth $S_{2} / J$. This inequality could be strict as shows Example 3.2 .

I am grateful to Professor Jürgen Herzog for useful discussions and comments during the preparation of the paper.

\section{The Behavior of DePth AND SDEPth Under REDUCTION MODUlo ELEMENT}

In dimension theory it is well known the following result (see e.g. [3, Proposition A.4], or [6, Corollary 10.9])

Theorem 1.1. If $(R, m)$ is a Noetherian local ring and $M$ is finitely generated $R$-module, then for any $x \in m$ we have

$$
\operatorname{dim} M / x M \geq \operatorname{dim} M-1 .
$$

If we consider reduction by a regular element, then the depth decreases by one. But what happens if we take reduction by a non-regular element?

Proposition 1.2. Let $S=K\left[x_{1}, \ldots, x_{n}\right]$ be a polynomial ring over a field $K, I \subset S$ a monomial ideal and $R=S / I$. Then

$$
\operatorname{depth}\left(R / x_{n} R\right) \geq \operatorname{depth}(R)-1 \text {. }
$$

Proof. Let $\bar{R}=R / x_{n} R \simeq S /\left(I, x_{n}\right)$. We denote $\bar{S}=K\left[x_{1}, \ldots, x_{n-1}\right]$ and let $x^{\prime}=$ $\left\{x_{1}, \ldots, x_{n-1}\right\}, x=\left\{x_{1}, \ldots, x_{n}\right\}$.

Let $\varphi$ be the canonical map from $R$ to $\bar{R}$ and $\alpha$ be the composite map

$$
\bar{S} \longrightarrow S \longrightarrow R=S / I,
$$

where the first map is the canonical embedding and the second map is the canonical surjection. It is clear that $\operatorname{ker}(\alpha)=I \cap \bar{S}$. Let $\alpha_{1}$ be the composite map

$$
\bar{S} \longrightarrow S \longrightarrow R=S / I \longrightarrow S /\left(I, x_{n}\right) \text {. }
$$

It is clear that $\alpha_{1}$ is surjective. We claim that $\operatorname{ker}\left(\alpha_{1}\right)=I \cap \bar{S}$. One inclusion is obvious. To prove other inclusion, we consider a monomial $v \in \operatorname{ker}\left(\alpha_{1}\right)$, that is, $v \in\left(I, x_{n}\right)$. Since $v \in \bar{S}$ and $I$ is a monomial ideal, it follows that $v \in I$. Let $\operatorname{ker}\left(\alpha_{1}\right)=\bar{I}$ then $\bar{S} / \bar{I} \simeq S /\left(I, x_{n}\right)$. It follows that the composition $\bar{R} \rightarrow R \rightarrow \bar{R}$ of the natural maps is the identity. Therefore, the $S$-module $\bar{R}$ is a direct summand of the $S$-module $R$. This implies that the $S$-module $H_{i}\left(x^{\prime} ; \bar{R}\right)$ is a direct summand of $H_{i}\left(x^{\prime} ; R\right)$ for all $i$, where $H_{i}\left(x^{\prime} ; \bar{R}\right)$ and $H_{i}\left(x^{\prime} ; R\right)$ are the i-th Koszul homology modules of $x^{\prime}$ with respect to $\bar{R}$ and $R$ respectively. In particular, if $H_{i}\left(x^{\prime} ; \bar{R}\right) \neq 0$, then $H_{i}\left(x^{\prime} ; R\right) \neq 0$. Let $k=\max \left\{i \mid H_{i}\left(x^{\prime}: \bar{R}\right) \neq 0\right\}$. Then $\operatorname{depth} \bar{R}=$ 
$n-1-k$, by [3, Theorem 1.6.17]. Since $H_{k}\left(x^{\prime} ; \bar{R}\right) \neq 0$, it follows that $H_{k}\left(x^{\prime} ; R\right) \neq 0$ which implies that $H_{k}(x ; R) \neq 0$ by [3, Lemma 1.6.18]. Therefore applying again [3, Theorem 1.6.17] it follows that $\operatorname{depth} R \leq n-k=\operatorname{depth} \bar{R}+1$.

Corollary 1.3. Let $I \subset S$ be a monomial ideal. Then $\operatorname{depth} S /(I: u) \geq \operatorname{depth} S / I$ for all monomials $u \notin I$.

Proof. Since $(I: u v)=((I: u): v)$, where $I$ is a monomial ideal and $u$ and $v$ are monomials, we may reduce to the case $u=x_{n}$, and apply recurrence. Then we have the exact sequence

$$
0 \longrightarrow S /\left(I: x_{n}\right) \longrightarrow S / I \longrightarrow S /\left(I, x_{n}\right) \longrightarrow 0 .
$$

By Depth Lemma [15, Lemma 1.3.9] and Proposition 1.2, we obtain the required result.

This Corollary does not hold (and so Proposition 1.2) if $u$ is not a monomial, as we have the following example:

Example 1.4. Let $S=K[x, y, z, t]$ and $I=(x, y) \cap(y, z) \cap(z, t)$ and $u=y+z$. Then $J:=(I: u)=(x, y) \cap(z, t)$ and $\operatorname{depth} S / J=1<2=\operatorname{depth} S / I$.

The Proposition 1.2 is not true in general. If $M$ is a finitely generated graded $R$-module and $x \in R_{1}$ then we might have

$$
\operatorname{depth}(M / x M)<\operatorname{depth}(M)-1,
$$

as shows the following example:

Example 1.5. Let $S=K[x, y, z, t], M=(x, y, z) /(x t)$. We have $\operatorname{depth} M=2$ and $M / x M=(x, y, z) /\left(x^{2}, x y, x z, x t\right)$. Since the maximal ideal is an associated prime ideal of $M / x M$, we get depth $M / x M=0$. Hence $\operatorname{depth}(M / x M)<\operatorname{depth}(M)-1$.

In Proposition 1.2 we might have

$$
\operatorname{depth}\left(R / x_{n} R\right)>\operatorname{depth}(R)-1,
$$

as shows the following example:

Example 1.6. Let $I=\left(x_{1}^{2}, x_{1} x_{2}, \ldots, x_{1} x_{n}\right) \subset S=K\left[x_{1}, \ldots, x_{n}\right]$ be a monomial ideal of $S$ and $R=S / I$. Then $\operatorname{depth}(R)=0$ since the maximal ideal $\left(x_{1}, x_{2}, \ldots, x_{n}\right) \in \operatorname{Ass}(R)$. Since $R / x_{1} R=S /\left(x_{1}\right) \simeq K\left[x_{2}, \ldots, x_{n}\right]$, we get $\operatorname{depth}\left(R / x_{1} R\right)=n-1$. Hence $\operatorname{depth}\left(R / x_{1} R\right)>$ $\operatorname{depth}(R)-1$.

For the sdepth we have a statement similar to that of Proposition 1.2. Indeed in the proof of [13, Lemma 1.2] where it was shown that sdepth $S /\left(I, x_{n}\right)=\operatorname{sdepth} S / I-1$ if $x_{n}$ is regular on $S / I$, we actually showed the following (without any assumption on $x_{n}$ ):

Lemma 1.7. Let $S=K\left[x_{1}, \ldots, x_{n}\right]$ be a polynomial ring over the field $K$. Let $I \subset S$ be any monomial ideal. Then

$$
\operatorname{sdepth}\left(S /\left(I, x_{n}\right)\right) \geq \operatorname{sdepth}(S / I)-1 .
$$

This lemma can not be extended to general multigraded modules $M$ as shows the following example, where the variable is even regular on $M$.

Example 1.8. Let $M=(x, y, z)$ be an ideal of $S=K[x, y, z]$. Consider a Stanley decomposition $M=z K[x, z] \oplus x K[x, y] \oplus y K[y, z] \oplus x y z K[x, y, z]$. Since sdepth $M \leq$ $\operatorname{dim} S=3$ and $M$ is not a principle ideal, it follows sdepth $M=2$. Note that $x$ induces a 
non-zero element in the socle of $M / x M$ which cannot be contained in any Stanley space of dimension greater or equal with one. Hence sdepth $M / x M=0$. Thus sdepth $M / x M<$ sdepth $M-1$.

However for the special case when $M$ is almost clean (see [9]), that is there exists a prime filtration $\mathcal{F}$ of $M$ such that $\operatorname{Supp}(\mathcal{F})=\operatorname{Ass}(M)$, we have the following:

Lemma 1.9. Let $M$ be a finitely generated multigraded $S$-module. If $M$ is almost clean and $x_{k} \in S$ is regular on $M$, then

$$
\text { sdepth } M / x_{k} M \geq \operatorname{sdepth} M-1 .
$$

Proof. Suppose that $\mathcal{F}$ is given by

$$
0=M_{0} \subset M_{1} \subset \ldots \subset M_{r}=M
$$

with $M_{i} / M_{i-1} \cong S / P_{i}\left(-a_{i}\right)$ for some $a_{i} \in \mathbb{N}^{n}$ and some monomial prime ideals $P_{i}$. Since Ass $M=\left\{P_{1}, \ldots, P_{r}\right\}$ and $x_{k}$ is regular on $M$, we get $x_{k} \notin P_{i}$ and so $x_{k}$ is regular on $M_{i} / M_{i-1}$. Set $\bar{M}_{i}=M_{i} / x_{k} M_{i}$. Then $\bar{M}_{i} \subset \bar{M}_{i+1}$ and $\left\{\bar{M}_{i}\right\}$ define a filtration $\overline{\mathcal{F}}$ of $\bar{M}=\bar{M}_{r}$ with $\bar{M}_{i} / \bar{M}_{i-1} \cong S /\left(P_{i}, x_{k}\right)\left(-a_{i}\right)$. Thus $\operatorname{sdepth} \bar{M} \geq \min _{i} \operatorname{dim} S /\left(P_{i}, x_{k}\right)=$ sdepth $M-1$ (see Corollary 2.3).

The above Example 1.8 hints that if $x$ is a regular element on $M$, then sdepth $M / x M \leq$ sdepth $M-1$. This is the subject of our next proposition.

Proposition 1.10. Let $M$ be finitely generated $\mathbb{Z}^{n}$-graded $S$-module and let $x_{k}$ be regular on $M$. If $\mathcal{D}_{1}: M / x_{k} M=\bigoplus_{i=1}^{r} \bar{m}_{i} K\left[Z_{i}\right]$, is a Stanley decomposition of $M / x_{k} M$, where $m_{i} \in M$ is homogeneous and $\bar{m}_{i}=m_{i}+x_{k} M$. Then

$$
M=\bigoplus_{i=1}^{r} m_{i} K\left[Z_{i}, x_{k}\right]
$$

is a Stanley decomposition of $M$. In particular

$$
\text { sdepth } M / x_{k} M \leq \operatorname{sdepth} M-1 .
$$

Proof. Let $N=\sum_{i=1}^{r} m_{i} K\left[Z_{i}, x_{k}\right]$. Then $N \subseteq M$. Since $\mathcal{D}_{1}$ is a Stanley decomposition of $M / x_{k} M$ it follows that $\psi(N)=M / x_{k} M$ where $\psi: M \rightarrow M / x_{k} M$ is the canonical epimorphism. This implies that $M=x_{k} M+N$ as $\mathbb{Z}^{n}$-graded $K$ vector spaces. We show that $M=N$. First we observe that $M=x_{k}^{d} M+N$ for all $d$. This follows by induction on $d$, because if we have $M=x_{k}^{d-1} M+N$, then $M=x_{k}^{d-1}\left(x_{k} M+N\right)+N=$ $x_{k}^{d} M+x_{k}^{d-1} N+N=x_{k}^{d} M+N$ since $x_{k}^{d-1} N \subset N$. This completes the induction. Since $M$ is finitely generated there exists an integer $c$ such that $\operatorname{deg}_{x_{k}}(m) \geq c$ for all homogeneous elements $m \in M$. Now let $m \in M$ be a homogeneous element with $\operatorname{deg}_{x_{k}}(m)=a$ and let $d>a-c$ be an integer. Since $M=x_{k}^{d} M+N$, there exist homogeneous elements $v \in M$ and $w \in N$ such that $m=x_{k}^{d} v+w$, where $a=\operatorname{deg}_{x_{k}} v+d=\operatorname{deg}_{x_{k}} w$. It follows that $\operatorname{deg}_{x_{k}} v=a-d<c$, a contradiction. It implies that $v=0$, hence $m=w \in N$.

Now we show that the sum $\sum_{i=1}^{r} m_{i} K\left[Z_{i}, x_{k}\right]$ is direct, that is

$$
m_{i} K\left[Z_{i}, x_{k}\right] \cap \sum_{\substack{j=1 \\ j \neq i}}^{r} m_{j} K\left[Z_{j}, x_{k}\right]=(0) .
$$

Let $u=m_{i} q_{i}=\sum_{\substack{j=1 \\ j \neq i}}^{r} m_{j} q_{j} \in M$ be homogeneous for some $q_{j}$ monomials in $K\left[Z_{j}, x_{k}\right]$ such that $\operatorname{deg}(u)=\operatorname{deg}\left(m_{j} q_{j}\right)$ for all $j$. Let $p$ be the biggest power of $x_{k}$ dividing $q_{i}$. If 
$p=0$, then we have $\bar{u}=\bar{m}_{i} q_{i} \neq 0$ in $M / x_{k} M$ since $\bar{m}_{i} K\left[Z_{i}\right]$ is a Stanley space. It follows that $\bar{u} \in \bar{m}_{i} K\left[Z_{i}\right] \cap \sum_{\substack{j=1 \\ j \neq i}}^{r} \bar{m}_{j} K\left[Z_{j}\right]$, a contradiction. In the case of $p>0$, then in $M / x_{k} M$ we get $\bar{u}=0=\sum_{\substack{j=1 \\ j \neq i}}^{r} \bar{m}_{j} \bar{q}_{j}$. It follows that $\bar{q}_{j}=0$, since $\mathcal{D}_{1}$ is a Stanley decomposition of $M / x_{k} M$. Thus $q_{j}=x_{k} q_{j}^{\prime}$ for some $q_{j}^{\prime} \in K\left[Z_{j}, x_{k}\right]$ and we get $x_{k}\left(m_{i} q_{i}^{\prime}-\sum_{\substack{j=1 \\ j \neq i}}^{r} m_{j} q_{j}^{\prime}\right)=0$, which implies $m_{i} q_{i}^{\prime}-\sum_{\substack{j=1 \\ j \neq i}}^{r} m_{j} q_{j}^{\prime}=0$ since $x_{k}$ is regular on $M$. Applying the same argument by recurrence we get $q_{j}=x_{k}^{p} s_{j}$ for some $s_{j} \in K\left[Z_{j}, x_{k}\right]$, and $m_{i} s_{i}=\sum_{\substack{j=1 \\ j \neq i}}^{r} m_{j} s_{j}$. We set $v=m_{i} s_{i}$. Since $\bar{s}_{i} \neq 0$, we get $\bar{v} \neq 0$ because $\bar{m}_{i} K\left[Z_{i}\right]$ is a Stanley space. On the other hand $\bar{v} \in \bar{m}_{i} K\left[Z_{i}\right] \cap \sum_{\substack{j=1 \\ j \neq i}}^{r} \bar{m}_{j} K\left[Z_{j}\right]$. It implies that $\bar{v}=0$, a contradiction.

Finally we show that each $m_{i} K\left[Z_{i}, x_{k}\right]$ is a Stanley space. Indeed, suppose that $m_{i} f=0$ for some $f \in K\left[Z_{i}, x_{k}\right]$ where $f=\sum_{j=0}^{a} f_{j} x_{k}^{j}$ such that $x_{k}$ does not divide $f_{j}$ for all $j$ then $\sum_{j=0}^{a} m_{i} f_{j} x_{k}^{j}=0$ implies that $\bar{m}_{i} f_{0}=0$ in $M / x_{k} M$. We get $f_{0}=0$ since $\bar{m}_{i} K\left[Z_{i}\right]$ is a Stanley space. It follows that $f=x_{k} g$ where $g=\sum_{j=1}^{a} f_{j} x_{k}^{j-1}$ and from $x_{k} m_{i} g=m_{i} f=0$ we get $m_{i} g=0, x_{k}$ being regular on $M$. Then induction on the degree of $f$ concludes the proof since $\operatorname{deg}_{x_{k}} g<\operatorname{deg}_{x_{k}} f$.

Corollary 1.11. If Stanley's conjecture holds for the module $M / x_{i} M$, where $x_{i} \in S$ is regular on $M$, then it also holds for $M$.

Corollary 1.12. The equality holds in Lemma 1.7, if $x_{n}$ is regular on $S / I$.

The proof follows from Lemma 1.7 and Proposition 1.10 for $M=S / I$.

Corollary 1.13. Let $\operatorname{depth} M=t$. If there exists $u=u_{1}, \ldots, u_{t} \in M o n(S)$ such that $u$ is regular sequence on $M$ then Stanley's conjecture holds for $M$.

Proof. For any regular sequence $u=u_{1}, \ldots, u_{t} \in \operatorname{Mon}(S)$ we may choose $u$ such that $u_{i}=x_{i_{j}}$ for all $1 \leq i \leq t$, where $x_{i_{j}} \in \operatorname{supp}\left(u_{i}\right)$, since for any monomial $u_{i} \in S$ being regular on $M$ implies that each $x_{i_{j}} \in \operatorname{supp}\left(u_{i}\right)$ is regular on $M$, because if $x_{i_{j}}$ belong to the set of zero divisors of $M$ then $x_{i_{j}} \in P$ for some $P \in \operatorname{Ass}(M)$, so $u_{i} \in P$, which is not true as $u_{i}$ is regular on $M$. Since $u$ is a maximal regular sequence on $M$, we have depth $M /\left(u_{1}, \ldots, u_{t}\right) M=0$. Applying Proposition 1.10 by recurrence we get $\operatorname{sdepth} M \geq$ sdepth $M /\left(u_{1}, \ldots, u_{t}\right) M+t \geq t=\operatorname{depth} M$. Hence Stanley's conjecture holds for $M$.

Example 1.14. Let $S=K[x, y, z, t]$ and $M=(x, y, z) /(x y)$. Since $\operatorname{depth} M=2$ and $\{z, t\}$ is a $M$-regular sequence, we may apply Corollary 1.13 to see that Stanley's conjecture holds for $M$.

Theorem 1.15. Let $M$ be a finitely generated multigraded $S$-module. If $M$ is almost clean and $x_{k} \in S$ is regular on $M$, then

$$
\text { sdepth } M / x_{k} M=\operatorname{sdepth} M-1 .
$$

The proof follows from Lemma 1.9 and Proposition 1.10 .

Theorem 1.16. Let $M$ be a finitely generated multigraded $S$-module. If $M$ is almost clean and $u \in S$ is a monomial, which is regular on $M$, then $\operatorname{sdepth} M / u M \geq \operatorname{sdepth} M-1$.

Proof. Let $u=x_{i_{1}}^{a_{1}} \ldots x_{i_{t}}^{a_{t}}$. Since $u$ is regular on $M$, it follows that each $x_{i_{k}} \in \operatorname{supp}(u)$ is regular on $M$, where we denote by $\operatorname{supp}(u)$ the set of all variables $x_{j}$ such that $x_{j}$ divides 
the monomial $u$. We consider an ascending chain of submodules of $M$ between $u M$ and $M$ where two successive members of the chain are of the form

$$
x_{i_{1}}^{b_{1}} \cdots x_{i_{k}}^{b_{k}} \cdots x_{i_{t}}^{b_{t}} M \subset x_{i_{1}}^{b_{1}} \cdots x_{i_{k}}^{b_{k}-1} \cdots x_{i_{t}}^{b_{t}} M,
$$

and where $b_{i} \leq a_{i}$ for all $i=1, \ldots, t$.

We obtain

$$
x_{i_{1}}^{b_{1}} \cdots x_{i_{k}}^{b_{k}-1} \cdots x_{i_{t}}^{b_{t}} M / x_{i_{1}}^{b_{1}} \cdots x_{i_{k}}^{b_{k}} \cdots x_{i_{t}}^{b_{t}} M \simeq M / x_{i_{k}} M,
$$

since each $x_{i_{k}} \in \operatorname{supp}(u)$ is regular on $M$. Therefore Lemma 1.9 and Corollary 2.3 imply that

$$
\operatorname{sdepth}(M / u M) \geq \operatorname{sdepth}\left(M / x_{i_{k}} M\right)=\operatorname{sdepth} M-1 .
$$

\section{The BeHAVIOR OF SDEPTH ON SHORT EXACT SEQUENCE OF MULTiGRAdED MOdulES}

The following "Depth Lemma" is well-known.

Lemma 2.1. [15, Lemma 1.3.9] If

$$
0 \rightarrow U \rightarrow M \rightarrow N \rightarrow 0
$$

is a short exact sequence of modules over a local ring $R$, then

(a) If $\operatorname{depth} M<\operatorname{depth} N$, then $\operatorname{depth} U=\operatorname{depth} M$.

(b) If $\operatorname{depth} M>\operatorname{depth} N$, then $\operatorname{depth} U=\operatorname{depth} N+1$.

We will show that most of the statements of the "Depth Lemma" are wrong if we replace depth by sdepth. We first observe

Lemma 2.2. Let

$$
0 \rightarrow U \stackrel{f}{\rightarrow} M \stackrel{g}{\rightarrow} N \rightarrow 0
$$

be an exact sequence of finitely generated $\mathbb{Z}^{n}$-graded $S$-modules. Then

$$
\operatorname{sdepth} M \geq \min \{\operatorname{sdepth} U, \operatorname{sdepth} N\}
$$

Proof. Let $\mathcal{D}: \quad U=\bigoplus_{i=1}^{r} u_{i} K\left[Z_{i}\right]$ be a Stanley decomposition of $U$ with $\operatorname{sdepth}(\mathcal{D})=$ sdepth $U$ and let $\mathcal{D}^{\prime}: N=\bigoplus_{j=1}^{s} n_{j} K\left[Z_{j}^{\prime}\right]$ be a Stanley decomposition of $N$ with $\operatorname{sdepth}\left(\mathcal{D}^{\prime}\right)$ $=\operatorname{sdepth} N$. Since $f$ is injective map, we may suppose that $f$ is an inclusion. Let $n_{j}^{\prime} \in M$ be a $\mathbb{Z}^{n}$ homogeneous element such that $g\left(n_{j}^{\prime}\right)=n_{j}$. Clearly, $M=\sum_{i=1}^{r} u_{i} K\left[Z_{i}\right]+$ $\sum_{j=1}^{s} n_{j}^{\prime} K\left[Z_{j}^{\prime}\right]$. We prove that the sum $\sum_{i=1}^{r} u_{i} K\left[Z_{i}\right]+\sum_{j=1}^{s} n_{j}^{\prime} K\left[Z_{j}^{\prime}\right]$ is direct. Set $V=\sum_{j} n_{j}^{\prime} K\left[Z_{j}^{\prime}\right]$. Since the exact sequence splits as linear spaces we see that $U \cap V=\{0\}$. Clearly $\mathcal{D}$ is already a Stanley decomposition of $U$ and remains to show only that if $y \in n_{j}^{\prime} K\left[Z_{j}^{\prime}\right] \cap \sum_{\substack{k=1 \\ k \neq j}}^{s} n_{k}^{\prime} K\left[Z_{k}^{\prime}\right]$ then $y=0$. As $g(y) \in n_{j} K\left[Z_{j}^{\prime}\right] \cap \sum_{\substack{k=1 \\ k \neq j}}^{s} n_{k} K\left[Z_{k}^{\prime}\right]=\{0\}$, we see that $y \in U$, that is $y \in U \cap V=\{0\}$.

Corollary 2.3. Let

$$
(0)=M_{0} \subset M_{1} \subset \ldots \subset M_{r-1} \subset M_{r}=M
$$

be an ascending chain of $\mathbb{Z}^{n}$-graded submodules of $M$. Then

$$
\operatorname{sdepth} M \geq \min \left\{\operatorname{sdepth} M_{i} / M_{i-1}: i \in\{1, \ldots, r\}\right\}
$$

for all $i \in[r]$. 
Proof. We consider the exact sequence of $\mathbb{Z}^{n}$-graded submodules of $M$ such that

$$
0 \rightarrow M_{i-1} \rightarrow M_{i} \rightarrow M_{i} / M_{i-1} \rightarrow 0 .
$$

By Lemma 2.2, we get sdepth $M_{i} \geq \min \left\{\operatorname{sdepth} M_{i-1}\right.$, sdepth $\left.M_{i} / M_{i-1}\right\}$. We apply induction to prove the inequality (3). For $i=1$ this holds clearly. We suppose (3) is true for $i=t$ then we have

$$
\operatorname{sdepth} M_{t} \geq \min \left\{\operatorname{sdepth} M_{i} / M_{i-1}: i \in\{1, \ldots, t\}\right\} .
$$

Let $i=t+1$ then we have $\operatorname{sdepth} M_{t+1} \geq \min \left\{\operatorname{sdepth} M_{t}\right.$, sdepth $\left.M_{t+1} / M_{t}\right\}$, which is enough.

The analogue of Lemma 2.1 (a) only holds under an additional assumption.

Corollary 2.4. In the hypothesis of Lemma 2.2 suppose that $\operatorname{sdepth} M<\operatorname{sdepth} N$. Then sdepth $M \geq \operatorname{sdepth} U$.

Proof. If sdepth $M<\operatorname{sdepth} U$, we get $\operatorname{sdepth} M<\min \{\operatorname{sdepth} U, \operatorname{sdepth} N\}$ contradicting Lemma 2.2 ,

The analogue of $2.1(b)$ is wrong.

Example 2.5. Let $S=K[x, y, z], M=(x, y, z)$. In the exact sequence $0 \rightarrow M \rightarrow S \rightarrow$ $K \rightarrow 0$, we have $\operatorname{sdepth} S=3>\operatorname{sdepth} K=0$ but sdepth $M=2 \neq \operatorname{sdepth} K+1$.

Note that the case treated in Proposition 1.10, that is the short exact sequence $0 \rightarrow$ $M \stackrel{x_{k}}{\rightarrow} M \rightarrow M / x_{k} M \rightarrow 0$, and Lemma 2.7 apparently hints that some analogue of $(b)$ from "Depth Lemma" in the frame of sdepth might be true. Unfortunately, this is not the case as shows the following:

Example 2.6. We have a resolution $0 \rightarrow \Omega^{1} m \rightarrow S^{3} \rightarrow m \rightarrow 0$, where $S=K[x, y, z]$ and $m=(x, y, z)$. Then $\Omega^{1} m$ is not free because otherwise $\operatorname{proj} \operatorname{dim}_{S} m$ should be 1 , which is not true. If sdepth $\Omega^{1} m=3$ then follows $\Omega^{1} m$ free by the elementary Lemma 2.9. Thus sdepth $\Omega^{1} m \leq 2=\operatorname{sdepth} m$.

However it remains still the problem in general that if for an exact sequence $0 \rightarrow U \rightarrow$ $M \rightarrow N \rightarrow 0, \operatorname{sdepth} M>\operatorname{sdepth} N$ implies $\operatorname{sdepth} U \geq \operatorname{sdepth} N+1$. In general this is false (see Example 2.6) but we prove this result in a special case.

Lemma 2.7. If $I \subset S=K\left[x_{1}, \ldots, x_{n}\right]$ is a monomial complete intersection, then sdepth $I$ $\geq \operatorname{sdepth} S / I+1$.

Proof. Let $\left\{v_{1}, \ldots, v_{m}\right\}$ be the regular sequence of monomials generating $I$. Since sdepth $S / I$ $=n-m$, by applying [13, Theorem 1.1] recursively, and sdepth $I \geq n-m+1$, by [7], or [9. Proposition 3.4], it follows the desired result.

In general for any monomial ideal the inequality in above lemma is still an open question. This inequality motivates that sdepth $I \geq \operatorname{sdepth} J / I+1$ for any two monomial ideals $I \subset J \subset S$. But this inequality does not hold as shows the following example:

Example 2.8. Let $S=K[x, y], I=\left(x y, y^{2}\right), J=I+\left(x^{2}\right)$. Then we have $\operatorname{sdepth} J / I=$ $1=\operatorname{sdepth} I=\operatorname{sdepth} J$.

Lemma 2.9. If $M$ is multigraded $S$-module, $S=K\left[x_{1}, \ldots, x_{n}\right]$ with sdepth $M=n$ then $M$ is free.

Proof. If sdepth $M=n$, then we have a Stanley decomposition of the form $M=\oplus_{i} u_{i} S$ and $u_{i} S$ are free $S$-modules. The direct sum is of linear spaces but it turns out to be of free $S$-modules. 


\section{The BeHAVIOR OF SDEPTH ON ALGEBRA TENSOR PRODUCT}

Theorem 3.1. Let $I \subset S_{1}=K\left[x_{1}, \ldots, x_{n}\right], J \subset S_{2}=K\left[y_{1}, \ldots, y_{m}\right]$ be monomial ideals and $S=K\left[x_{1}, \ldots, x_{n}, y_{1}, \ldots, y_{m}\right]$. Then $\operatorname{sdepth} S_{1} / I+\operatorname{sdepth} S_{2} / J \leq \operatorname{sdepth} S /(I S, J S)$.

Proof. Let

$$
\mathcal{D}_{1}: S_{1} / I=\bigoplus_{i=1}^{r} u_{i} K\left[Z_{i}\right]
$$

be a Stanley decomposition of $S_{1} / I$ such that $\operatorname{sdepth} \mathcal{D}_{1}=\operatorname{sdepth} S_{1} / I$ and

$$
\mathcal{D}_{2}: S_{2} / J=\bigoplus_{j=1}^{s} v_{j} K\left[W_{j}\right]
$$

be a Stanley decomposition of $S_{2} / J$ such that $\operatorname{sdepth} \mathcal{D}_{2}=\operatorname{sdepth} S_{2} / J$. Then we have

$$
\begin{aligned}
S / I S & =S_{1}\left[y_{1}, \ldots, y_{m}\right] / I S \\
& =\left(S_{1} / I\right)\left[y_{1}, \ldots, y_{m}\right] \\
& =\bigoplus_{i=1}^{r} u_{i} K\left[Z_{i}\right]\left[y_{1}, \ldots, y_{m}\right] \\
& =\bigoplus_{i=1}^{r} u_{i} K\left[Z_{i}, y_{1}, \ldots, y_{m}\right]
\end{aligned}
$$

and

$$
\begin{aligned}
S / J S & =S_{2}\left[x_{1}, \ldots, x_{n}\right] / J S \\
& =\left(S_{2} / J\right)\left[x_{1}, \ldots, x_{n}\right] \\
& =\bigoplus_{j=1}^{s} v_{j} K\left[W_{j}\right]\left[x_{1}, \ldots, x_{n}\right] \\
& =\bigoplus_{j=1}^{s} v_{j} K\left[W_{j}, x_{1}, \ldots, x_{n}\right]
\end{aligned}
$$

We claim that

$$
S /(I S, J S)=\bigoplus_{i, j} u_{i} v_{j} K\left[Z_{i}, W_{j}\right]
$$

Let $w \in(I S, J S)^{c}=S /(I S, J S)$ be a monomial; that is, $w \in S$ and $w \notin(I S, J S)$. We have $w \notin I S$ and $w \notin J S$. It follows that $w \in(I S)^{c}$ and $w \in(J S)^{c}$. Hence there exist $i$ and $j$ such that $w \in u_{i} K\left[Z_{i}, y_{1}, \ldots, y_{m}\right]$ and $w \in v_{j} K\left[W_{j}, x_{1}, \ldots, x_{n}\right]$. So we have $w \in$ $u_{i} K\left[Z_{i}, y_{1}, \ldots, y_{m}\right] \cap v_{j} K\left[W_{j}, x_{1}, \ldots, x_{n}\right]$ and $u_{i} K\left[Z_{i}, y_{1}, \ldots, y_{m}\right] \cap v_{j} K\left[W_{j}, x_{1}, \ldots, x_{n}\right]=$ $u_{i} v_{j} K\left[Z_{i}, W_{j}\right]$, since $u_{i} \in S_{1}$ and $v_{j} \in S_{2}$.

In order to prove the opposite inclusion, consider a monomial $v \in u_{i} v_{j} K\left[Z_{i}, W_{j}\right]$. Then $v \in u_{i} K\left[Z_{i}, y_{1}, \ldots, y_{n}\right] \subset(I S)^{c}$ and similarly $v \in(J S)^{c}$. Thus $v \in(I S, J S)^{c}$. So $S /(I S, J S)=\sum_{i, j} u_{i} v_{j} K\left[Z_{i}, W_{j}\right]$.

Now we prove that this sum is direct. Let $i_{1}, i_{2} \in[r]$ and $j_{1}, j_{2} \in[s]$ be such that $\left(i_{1}, j_{1}\right) \neq\left(i_{2}, j_{2}\right)$, let us say $i_{1} \neq i_{2}$. Then $u_{i_{1}} v_{j_{1}} K\left[Z_{i_{1}}, W_{j_{1}}\right] \cap u_{i_{2}} v_{j_{2}} K\left[Z_{i_{2}}, W_{j_{2}}\right] \subset$ $u_{i_{1}} K\left[Z_{i_{1}}, y_{1}, \ldots, y_{m}\right] \cap u_{i_{2}} K\left[Z_{i_{2}}, y_{1}, \ldots, y_{m}\right]=\{0\}$, which shows our claim. It follows that sdepth $S_{1} / I+\operatorname{sdepth} S_{2} / J \leq \operatorname{sdepth} S /(I S, J S)$. 
The following example shows that the inequality in the above theorem can be strict.

Example 3.2. Let $S=K\left[x_{1}, x_{2}, x_{3}, x_{4}, x_{5}, x_{6}, x_{7}, x_{8}\right]$ be a polynomial ring over the field $K$. Let $I=\left(x_{1} x_{3}, x_{1} x_{4}, x_{2} x_{3}, x_{2} x_{4}\right) \subset S_{1}=K\left[x_{1}, x_{2}, x_{3}, x_{4}\right]$ be the ideal of the polynomial ring $S_{1}$ and $J=\left(x_{5} x_{7}, x_{5} x_{8}, x_{6} x_{7}, x_{6} x_{8}\right) \subset S_{2}=K\left[x_{5}, x_{6}, x_{7}, x_{8}\right]$ be the ideal of the polynomial ring $S_{2}$. Consider the ideal $(I S, J S) \subset S$, then a Stanley decomposition $\mathcal{D}$ of $S /(I S, J S)$ is

$\mathcal{D}: S /(I S, J S)=K\left[x_{1}, x_{2}, x_{5}\right] \oplus x_{3} K\left[x_{3}, x_{5}, x_{6}\right] \oplus x_{4} K\left[x_{4}, x_{5}, x_{6}\right] \oplus x_{6} K\left[x_{1}, x_{2}, x_{6}\right] \oplus$ $x_{7} K\left[x_{1}, x_{2}, x_{7}\right] \oplus x_{8} K\left[x_{1}, x_{2}, x_{8}\right] \oplus x_{3} x_{4} K\left[x_{3}, x_{4}, x_{5}\right] \oplus x_{3} x_{7} K\left[x_{3}, x_{7}, x_{8}\right] \oplus x_{3} x_{8} K\left[x_{3}, x_{4}, x_{8}\right] \oplus$ $x_{4} x_{7} K\left[x_{3}, x_{4}, x_{7}\right] \oplus x_{4} x_{8} K\left[x_{4}, x_{7}, x_{8}\right] \oplus x_{5} x_{6} K\left[x_{1}, x_{5}, x_{6}\right] \oplus x_{7} x_{8} K\left[x_{1}, x_{7}, x_{8}\right]$ $\oplus x_{2} x_{5} x_{6} K\left[x_{1}, x_{2}, x_{5}, x_{6}\right] \oplus x_{3} x_{4} x_{6} K\left[x_{3}, x_{4}, x_{5}, x_{6}\right] \oplus x_{2} x_{7} x_{8} K\left[x_{1}, x_{2}, x_{7}, x_{8}\right]$ $\oplus x_{3} x_{4} x_{7} x_{8} K\left[x_{3}, x_{4}, x_{7}, x_{8}\right]$, hence $\operatorname{sdepth} \mathcal{D}=3$. Note that sdepth $S_{1} / I=1$ with a Stanley decomposition $S_{1} / I=K\left[x_{1}, x_{2}\right] \oplus x_{3} K\left[x_{3}\right] \oplus x_{4} K\left[x_{3}, x_{4}\right]$. We observe that $\operatorname{sdepth} S_{1} / I$ can not be greater than one. Similarly we have sdepth $S_{2} / J=1$. Hence we obtain that $\operatorname{sdepth} S_{1} / I+\operatorname{sdepth} S_{2} / J<\operatorname{sdepth} S /(I S, J S)$.

The following corollary is a particular case of [13, Theorem 1.1].

Corollary 3.3. Let $I \subset S$ be a monomial ideal and $u \in S$ is a monomial, which is regular on $S / I$. Then $\operatorname{sdepth} S /(I, u) \geq \operatorname{sdepth} S / I-1$.

Proof. Renumbering $x_{i} \in \operatorname{supp}\left(u_{j}\right)$ for all $u_{j} \in G(I)$, we may suppose that $I$ is generated by a monomial ideal $J \subset S_{1}=K\left[x_{1}, \ldots, x_{r}\right]$ and $u \in S_{2}=K\left[x_{r+1}, \ldots, x_{n}\right]$ for some $1<r<n$. Then $\operatorname{sdepth} S /(I, u) \geq \operatorname{sdepth} S_{1} / J+\operatorname{sdepth} S_{2} /(u)$, by Theorem 3.1. Since sdepth $S_{2} /(u)=n-r-1$ and $\operatorname{sdepth} S / I=\operatorname{sdepth} S_{1} / J+n-r$, by [13, Lemma 1.2], it follows that $\operatorname{sdepth} S /(I, u) \geq \operatorname{sdepth} S / I-1$.

In the analogue of Theorem 3.1 for depth we have equality, that is $\operatorname{depth} S /(I S, J S)=\operatorname{depth} S_{1} / I+\operatorname{depth} S_{2} / J$ (see [15, Theorem 2.2.21]). We note that if Stanley's conjecture hold for the modules $S_{1} / I$ and $S_{2} / J$ it holds also for $S /(I S, J S)$.

\section{REFERENCES}

[1] I. Anwar, Janet's algorithm, Bull. Math. Soc. Sc. Math. Roumanie 51(99), (2008), 11-19.

[2] I. Anwar, D. Popescu, Stanley conjecture in small embedding dimension, J. Alg., 318 (2007), 10271031 .

[3] W. Bruns, J. Herzog, Cohen Macaulay Rings, Revised Edition, Cambridge: Cambridge University Press, 1996.

[4] M. Cimpoeas, Stanley depth of complete intersection monomial ideals, Bull. Math. Soc. Sc. Math. Roumanie, 51(99)(2008), 205-211.

[5] M. Cimpoeas, Stanley depth of monomial ideals in three variables. arXiv:math. AC/0807.2166, preprint (2008).

[6] D. Eisenbud, Commutative algebra; with a view towards algebraic geometry, Graduate Text in Maths., New York: Springer-Verlang, 1995.

[7] J. Herzog, A. S. Jahan, S. Yassemi, Stanley decompositions and partitionable simplicial complexes. J. Alg. Comb., 27(1), (2007), 113-125.

[8] J. Herzog, D. Popescu, Finite filtrations of modules and shellable multicomplexes, Manuscripta Math., 121 (2006), 385-410.

[9] J. Herzog, M. Vladoiu, X. Zheng, How to compute the Stanley depth of a monomial ideal, J. Alg., in press.

[10] A. S. Jahan, Prime filtrations of monomial ideals and polarizations, J. Alg., 312(2), (2007), 1011-1032.

[11] S. Nasir, Stanley decompositions and localization, Bull. Math. Soc. Sc. Math. Roumanie, 51(99), (2008), 151-158.

[12] D. Popescu, Stanley depth of multigraded modules, arXiv:math. AC/0801.2632, preprint (2008). 
[13] A. Rauf, Stanley Decompositions, Pretty Clean Filtrations and Reductions Modulo Regular Elements, Bull. Math. Soc. Sc. Math. Roumanie, 50(98), (2007), 347-354.

[14] R. P. Stanley, Linear Diophantine Equations and Local Cohomology, Invent. Math., 68 (1982), 175193.

[15] R. H. Villareal, Monomial Algebras, New York: Marcel Dekker Inc, (2001).

Asia Rauf, Abdus Salam School of Mathematical Sciences, GC University, Lahore

E-mail address: asia.rauf@gmail.com 